Results Violence - physical assault, sexual assault, drink-driving crashes, and suicide - comprised $84 \%$ of the burden in Alexandra, $74 \%$ in Zacatecas, $65 \%$ in Leuven, and 56\%-59\% elsewhere. Physical and sexual assault alone comprised 32\%-72\%. Drink-driving comprised 10\%-21\%.

Conclusions Achieving this SDG goal will require creating effective violence prevention programs. Our physical assault estimates greatly exceed GBD's estimates. GBD's assault incidence sets a high severity threshold for qualifying cases. Its alcohol-attributable fractions for physical assault average one third of the estimates in widely respected multi-national studies. GBD 2017 also attributed no sexual violence to harmful alcohol use.

\section{C.003 DRINK SPECIAL LAWS AND ALCOHOL-RELATED FATAL CRASHES IN THE UNITED STATES}

1,2Victor Puac-Polanco*, ${ }^{2}$ Pia Mauro, ${ }^{2}$ Katherine Keyes, ${ }^{2}$ Charles Branas. ${ }^{1}$ Harvard Medical School Department of Health Care Policy, Boston, USA; ${ }^{2}$ Columbia University Department of Epidemiology, New York, USA

10.1136/injuryprev-2021-safety. 176

Background In the United States, every day, close to 30 people die in alcohol-impaired related crashes. Given the known role of alcohol in traffic injuries, we assessed the impact of drink special laws on alcohol- and non-alcohol-related fatal motor vehicle crash rates.

Methods We performed a synthetic control analysis of US data from 1982 to 2017 . We used publicly available data to evaluate the effects of implementing any drink special law at the state-level during the study period on fatality rates per 100 million vehicle-miles of travel. We used an enhanced version of the SC method for the analysis of multiple treated units.

Results Overall, treated states with any drink special law reduced alcohol-related fatal crashes per 100 million VMT by $0.01(\mathrm{p}$-value $=0.84)$ in year one, $0.10(\mathrm{p}$-value $=0.14)$ in year three, 0.07 ( $\mathrm{p}$-value $=0.28$ ) in year five, and $0.16(\mathrm{p}$ value $=0.01$ ) in year 10 post-implementation compared to the synthetic control trend. Implementation of any drink special law also produced reductions of non-alcohol-related fatal crashes per 100 million VMT by 0.19 (p-value $=0.11)$ in year one, 0.24 ( $\mathrm{p}$-value $=0.03)$ in year three, 0.25 (p-value $=0.00)$ in year five, and $0.18(\mathrm{p}$-value $=0.00)$ in year 10 compared to the synthetic control trend. Findings for the number of laws implemented and each drink special laws were mixed.

Conclusions Drink special laws appeared to be associated with larger reductions of non-alcohol-related fatal crashes but no with the hypothesized target population, the alcohol-related fatal crashes.

\section{D - Road - Alcohol, March 25, 2021}

\section{D.001 DRINK DRIVING - WHY WE OFTEN FAIL TO LEARN FROM BEST PRACTICE}

Matej Košir*, Sanela Talić. Institute For Research and Development 'Utrip', Borovnica, Slovenia

10.1136/injuryprev-2021-safety. 177
In Europe, approximately $25 \%$ of all traffic accident fatalities are alcohol-related. Governments and other responsible authorities have carried out a number of more or less effective and (also) ineffective measures to prevent drink driving. Some policies and measures are proven to be successful (e.g. more frequent random BAC tests for all drivers, zero tolerance for novice and professional drivers, lower BAC levels in general, alcolocks for repeat offenders, structured and long-term campaigns and educational programmes etc.), but are still not fully included in many legislations and are not consistently implemented in practice. Despite the fact that drivers today are aware of the tragic and financial consequences they can bring to themselves and others while driving drunk, and that the public opinion with regard to drink driving largely changed (most Europeans nowadays openly oppose to drink driving), drunk drivers are still responsible for about a quarter of all fatal accidents in Europe. Therefore, new, better and more effective measures are urgently needed. The authors will present an extensive existing scientific evidence from many studies and research in a structured way and present many good examples from practice as well (e.g. evidence-based interventions). Presentation will be based on the document (guidelines and recommendations) which was recently published by author (Košir) and co-author (Talić) in Slovenia and co-sponsored by the Slovenian Traffic Safety Agency and the Ministry of Health. Link to the document: https://goo.gl/ cU4zWg.

\section{E - intentional, March 25, 2021}

\section{E.001 IF WE DON'T ASK, WE DON'T KNOW AND WE CAN'T HELP}

Mariana Galrao*, Richelle Douglas, Alison Creagh, Sarah Smith. Sexual Health Quarters, Northbridge, Australia

10.1136/injuryprev-2021-safety. 178

Context In Australia, one woman is murdered by a partner every 9 days and 26,000 children are homeless every year due to domestic violence. Consumer-centred, cost-effective violence prevention programs are urgently needed.

Research indicates those exposed to violence want to be asked about it. However, clinicians find it too awkward and too time-consuming. As a result, questions are not asked and the exposure to violence continues. Our aim is to design and implement an innovative screening program for partner violence that is acceptable to both consumers and health staff, transferable to other settings and sustainable into the future.

Process 1) developed screening and risk assessment tools, validated by consumers and health staff; 2) developed referral pathways; 3) trained staff; 4) modified clinic layout to be consumer-centred; 5) implemented program at Sexual Health Quarters; 6) collected staff and consumer feedback before and after implementation.

Analysis quantitative analysis of 1) prevalence of violence; 2) characteristics of survivors; 3) support required; 4) staff and consumer feedback.

Outcomes In the first 6 months of the program we screened 1500 women, identified exposure to violence in $18 \%$ and provided counselling to $40 \%$ of those exposed. All consumers and staff surveyed supported this program, with $90 \%$ of staff admitting that it was easier than expected. 